\title{
Curriculare Verankerung des Forschungsnahen Lernens
}

\section{Ludwig Huber ${ }^{\dagger}$}

Forschendes Lernen zeichnet sich durch eine Vielfalt unterschiedlicher Formen und Ausgestaltungen aus. Für eine nachhaltige und langfristige Verankerung solcher Ansätze stellt sich die Frage, welche Möglichkeiten sich innerhalb der Curricula der Studiengänge dafür bieten. Der Beitrag stellt verschiedene Formen der Verankerung vor und diskutiert ihre Eignung.

\section{$1 \quad$ Ausgangslage und Fragestellung}

In den letzten Jahren sind in der Lehre an den Hochschulen Deutschlands in großer Menge und Vielfalt innovative Versuche mit Lehrveranstaltungen und Projekten unter der Devise des Forschenden, Forschungsorientierten oder Forschungsbasierten Lernens unternommen worden. Aus Gründen, die anderswo ausführlicher abgehandelt worden sind (Huber, 2014), werden im Folgenden alle diese Ansätze unter der Bezeichnung „Forschungsnahes Lernen“ zusammengefasst. Mit diesem Begriff ist die im Grundsatz gemeinsame Intention dieser Ansätze gut bezeichnet, während die konkreten Ausgestaltungen der Veranstaltungen und Projekte stark variieren kann, sodass der Oberbegriff des „Forschenden Lernens“ hier nicht passend erscheint. 
So verschieden wie die Ausgestaltungen des Forschungsnahen Lernens sind nun allerdings auch bisher die Verhältnisse, in denen sie zu den Curricula, also den kodifizierten Vorgaben für die Studiengänge, stehen. Das Spektrum reicht von Veranstaltungen, Modulen, Projekten oder Phasen des Forschungsnahen Lernens, die als für alle verpflichtende Bestandteile in den Studiengangsordnungen enthalten sind, bis zu Projekten, die von Studierenden freiwillig (und zusätzlich) gewählt werden können. Vor allem aber ist der gegenwärtige Zustand dadurch gekennzeichnet, dass die Veranstaltungen des Forschungsnahen Lernens bisher zum größten Teil aus dem persönlichen Engagement einzelner Lehrender oder Lehrendenteams hervorgegangen sind. Dieses wiederum wurde und wird in Deutschland in den letzten Jahren wesentlich durch befristete Förderinstrumente (wie z. B. dem Qualitätspakt Lehre - QPL) oder durch Wettbewerbe stimuliert (wie z. B. im Rahmen der Exzellenz-Initiative), die etliche Hochschulen zu einer Profilierung auch durch eine „forschungsorientierte Lehre“ (o. Ä.) herausgefordert haben, oder schließlich auch durch hochschulinterne Fonds für solche Lehre gefördert. Das Ergebnis ist, dass die einzelnen Veranstaltungen des Forschungsnahen Lernens bisher meist nur punktuell und einmalig oder mit wenigen Wiederholungen angeboten werden und es noch ungewiss ist, inwieweit die Bemühungen nach Auslaufen jener (befristeten) Förderprogramme überhaupt fortgesetzt werden.

Wenn Forschungsnahes Lernen prinzipiell auch für die Zukunft erwünscht ist (wovon ich ausgehe), stellt sich angesichts dieser Situation die Frage, ob und wie dieses in einer Hochschule dauerhaft und regelmäßig angeboten werden soll und kann. Die Frage ist an sich so alt wie das Programm des „Forschenden Lernens“ - schon die Bundesassistentenkonferenz hat verschiedene mögliche Muster für dessen Verzahnung mit der Wissensvermittlung vorgestellt und erörtert (BAK, 1970, Textziffer 4.4). Sie ist aber angesichts der neuen Entwicklungen auch dringlich, nicht nur im Hinblick auf die jahrgangsübergreifende Planbarkeit des Studienangebots und seine grundsätzliche Verlässlichkeit für die Studierenden, sondern auch, weil Forschungsnahes Lernen an die Lehrenden hohe Anforderungen stellt, für die sie sich durch Weiterbildung und Austausch untereinander qualifizieren müssen, und weil es bei den Studierenden auf Voraussetzungen baut, die, je nach Format, in vorausgehenden Etappen vorbereitet werden müssen. Die Frage stellt sich auf verschiedenen Handlungsebenen der Hochschule: auf der ihres Leitbildes für die Hochschulbildung (oder auch: ihres Lehrprofils) und auf der des Curriculums für die Studierenden. Die curriculare Ebene soll hier im Mittelpunkt stehen, die Verankerung von Forschungsnahem Lernen im Leitbild der Hochschule aber zuvor noch wenigstens kurz beleuchtet werden. 


\section{Verankerung von Forschungsnahem Lernen im Lehrprofil der Hochschule}

Auf dieser Handlungsebene geht es um das nachhaltige institutionelle Engagement der Hochschule. Es kann sich in verschiedener Weise manifestieren, wie in den folgenden Unterkapiteln dargestellt.

\subsection{Deklaration von Forschungsnahem Lernen als didaktisches Profilmerkmal}

Die Hochschule oder ein Fachbereich proklamiert in Selbstbeschreibungen und Programmen Forschungsnahes Lernen (Forschendes, Forschungsorientiertes oder Forschungsbasiertes Lernen, die Begriffe dafür wechseln) als ein Prinzip, das ihre Lehre bestimmt bzw. bestimmen soll. Den Raum für solche Erklärungen bieten zumeist die Selbstdarstellungen auf den Homepages der Hochschulen (siehe die Universitäten Bremen und Oldenburg, das Karlsruher Institut für Technologie, die Fachhochschule Köln u.v. a.) oder Leitbilder, etwa in Anträgen im Rahmen der Exzellenzinitiative (siehe die Humboldt-Universität zu Berlin oder die Ludwig-Maximilians-Universität München).

Es liegt in der Gattung solcher Texte, dass sie zumeist nicht sogleich mitteilen, ob aus den Zielen konkrete Maßnahmen abgeleitet werden oder ob es den einzelnen Lehrenden überlassen bleibt, wie und in welchem Maße sie diese verfolgen. Damit wird Forschungsnahes Lernen zwar als didaktisches Prinzip formuliert, auf das man sich berufen kann, aber dessen konkrete Verankerung noch nicht gewährleistet. Eine solche Selbstverpflichtung kann, wenn durchgehalten, im günstigen Fall jedes Jahr erneut punktuelle Projekte anregen, ihnen aber nicht nachhaltig einen bestimmten Platz im Curriculum sichern.

\subsection{Einrichtung eines internen Förderprogramms für Forschungsnahes Lernen}

Die Hochschule (oder auch ein Fachbereich) richtet ein Förderprogramm ein, aus dem regelmäßig (auf Antrag) Projekte des Forschungsnahen Lernens gefördert oder Preise für Lehrende oder Studierende, die Forschungsnahes Lernen in herausragender Weise praktiziert haben, vergeben werden können.

Ein solches Programm für die interne Ausschreibung und Förderung existiert mittlerweile an etlichen Universitäten und Fachhochschulen in Deutschland und ist 
stärker als die programmatische Deklaration geeignet, Forschungsnahes Lernen als Profilmerkmal einer Hochschule dauerhaft auszuprägen. Darüber hinaus können solche Programme dauerhaft oder wechselnd Schwerpunkte setzen, die etwa auf die Entwicklung von Forschungsnahem Lernen in der Studieneingangsphase oder in Verbindung mit Laborarbeit oder Berufspraktika gerichtet sind und insofern auch einen möglichen Ort für sie im Curriculum bezeichnen. Die Formate des Forschungsnahen Lernens, die so gefördert werden sollen, können noch sehr verschieden sein: Sie reichen von veranstaltungsgebundenen Projekten bis zu freien von Studierenden betriebenen Forschungsvorhaben. Eine Verankerung in den Studiengängen ist damit allein noch nicht bewirkt.

\section{Verankerung des Forschungsnahen Lernens in Bildungsprogrammen und Curricula}

"Curriculum“ als pädagogischer Begriff, dies muss vorweg erklärt werden, kann in einer weiteren und in einer engeren Bedeutung verwendet werden: In der weiteren bezeichnet das Curriculum die planvolle Anordnung aller Lernsituationen, die Lernende in einem gegebenen räumlich-zeitlichen Rahmen in einer Schule oder Hochschule durchlaufen sollen, in der engeren die planvolle Sequenz der Lernsituationen in einem Fach oder Studiengang. In beiden Fällen umfasst das Curriculum - anders als "Lehrplan“ oder engl. „syllabus“ - nicht nur Ziele und Inhalte, sondern auch die Lehr- und Lernformen (Robinsohn, 1972) und damit auch ausdrücklich Formen des Forschungsnahen Lernens. In der weiten Bedeutung drückt „Curriculum" die Ziele, Wertvorstellungen und Arbeitsformen einer Bildungseinrichtung aus, die ihrem Bildungsangebot insgesamt, also über die einzelnen „Lehrgänge“ hinaus, zugrunde liegen, bezogen auf die Hochschule also ihre Vorstellung darüber, was Studieren in ihrem Rahmen eigentlich bedeuten soll. In diesem Bildungsprogramm insgesamt sollte Forschungsnahes Lernen (FnL) verankert sein. Im gängigen Sprachgebrauch wird jedoch unter „curricularer Verankerung des FnL“ verstanden, dass es in den Studiengängen, genauer: in deren Modulen, als Pflicht-, Wahl- oder Wahlpflichtveranstaltung vorgesehen und mit ECTS-Punkten ausgestattet ist. Frei wähl- bzw. organisierbare Projekte, für die es allenfalls irgendeine Form von Zertifikaten gibt, werden als „extracurricular“ bezeichnet (Reinmann, 2018, S. 6), obwohl sie im Bildungsprogramm der Hochschule einen bedeutenden Platz einnehmen können (s. u. 3.1). 
Im Folgenden wird zunächst ein Überblick über die möglichen Formen einer curricularen Verankerung gegeben, bevor grundsätzliche Probleme, die damit verbunden sind, erörtert werden.

\subsection{Forschungsnahes Lernen in Wahlveranstaltungen und Projekten außerhalb der Studiengänge}

In einer Hochschule können Lehrveranstaltungen mit Forschendem Lernen oder nicht an Veranstaltungen gebundene Projekte zumeist im überfachlichen Wahlbereich angeboten werden, für die kein fester verbindlicher Platz im Studiengang ausgewiesen ist. Sie gelten als Wahlveranstaltungen, für die Studierende sich je nach Vorgaben manchmal ECTS-Punkte anrechnen lassen oder aber mindestens eine Bescheinigung oder ein Zertifikat bekommen können (evtl. in einem Diploma supplement). Rückbindungen ans Curriculum sind, ähnlich wie beim Service Learning, möglich, wenn zwar nicht die Projekte, aber zum Beispiel Reflexionsseminare für solche Aktivitäten einen Platz im Studiengang haben.

Dies ist gewiss das Format mit den größten Freiheiten: Die Beteiligung daran ist für Lehrende wie für Studierende freiwillig, die Wahl der Fragestellungen und der Methoden offen, abhängig davon der Zeitpunkt und die Dauer frei entscheidbar.

Eine besondere Variante dieser Form stellen „Börsenprogramme“ dar, in deren Rahmen rein studentische Forschungsprojekte auch außerhalb von Lehrveranstaltungen gefördert oder Studierende, die solche Forschung machen wollen, mit Lehrenden, die sie in solchen Projekten fördern oder eigene Projekte mit ihnen zusammen durchführen wollen, zusammengebracht werden.

Das meines Wissens früheste Beispiel in Deutschland bot und bietet die Rheinisch-Westfälische Technische Hochschule Aachen, die sich ausdrücklich an die in den USA weitverbreiteten Formen des „Undergraduate Research Opportunity Program" (UROP) anschließt und auch mit renommierten amerikanischen Institutionen per Austausch zusammenarbeitet (RWTH Aachen University, 2019). Ein Weiteres ist das mit dem "Ars legendi-Preis“ der Hochschulrektorenkonferenz (HRK) ausgezeichnete Programm „Humboldt reloaded“ der Universität Hohenheim. Es ermöglicht forschungsnahes Lernen bereits im Grundstudium, im Prinzip außerhalb des vorgeschriebenen Studiengangs ihres Faches, jedoch im Wahlbereich anrechenbar. Die Studierenden arbeiten in kleinen Teams an Forschungsfragen; dafür bewerben sie sich auf von Lehrenden ausgeschriebene Projekte - meist ihrer Fakultät - oder können auch selbst solche ausschreiben und Betreuung dafür suchen (Universität Hohenheim, 2019). Weitere Beispiele bieten etwa die Humboldt-Uni- 
versität zu Berlin (dort in Verbindung mit Tutorien) und die Technische Universität Berlin, die Universität Göttingen und andere.

Solche Projekte kommen nur zustande, wenn Lehrende und Studierende ein eigenes Interesse daran haben und dafür auch den eventuell erhöhten Zeitaufwand und das Erfolgsrisiko auf sich nehmen. Sie können als elitär gelten, insofern sie nur die Studierenden mit entsprechender Initiative erreichen und aufgrund von Ausschreibung und Wettbewerb selegieren, eröffnen diesen aber besondere Chancen. Sie haben nicht die Verbindlichkeit einer in den Studiengang integrierten Veranstaltung und ihre Verknüpfung mit anderen, vorbereitenden oder nachfolgenden Teilen desselben bleibt ungewiss. Dafür bieten sie aber einen großen Spielraum für individuelle Interessen und die Entwicklung eigener Fragestellungen, die das Studium noch weiter begleiten könnten.

\subsection{Forschungsnahes Lernen in Verbindung mit Lehrveranstaltungen innerhalb eines Studiengangs}

Forschungsnahes Lernen in Verbindung mit Lehrveranstaltungen innerhalb eines Studienganges ist gegenüber der eben genannten Variante nach den vorliegenden Berichten bisher die bei Weitem häufigere Form der Verankerung. Diese Form wird in einer Vielzahl von Formaten und Muster in Studiengängen situiert (Huber, 2014; Stang, 2020). Im Folgenden wird die Art der Verankerung danach unterschieden, ob Forschungsnahes Lernen einmalig, wiederholt oder in sequenziellem Aufbau im Studiengang verortet wird.

\subsection{1 "Einmalig"}

Ein Modul oder eine Lehrveranstaltung innerhalb eines Moduls wird in der Studienordnung verbindlich für Forschungsnahes Lernen vorgesehen und ausgestattet (Lehrkapazität, Zeit, ECTS-Punkte). Und zwar nach Möglichkeit für Forschendes Lernen im engeren Sinne: Es handelt sich also um Projekte, in denen der Phasenzyklus des Forschens möglichst vollständig durchlaufen wird - mit den entsprechenden Herausforderungen bezüglich Zeitaufwand, Methodenvorbereitung und anderem. Die Wahl des Zeitpunkts im Studium kann dabei variieren: Angebote von Forschungsnahem Lernen können im Studienverlaufsplan zu Beginn (gleichsam als Initiation ins Studium), in der Mitte (vielleicht mit dem Schwerpunkt Methodenlernen verbunden) oder gegen Ende des Bachelor- oder Masterstudiums (evtl. schon als Vorbereitung der Abschlussarbeit) festgelegt werden. Alternativ gibt es auch die Möglichkeit, die Entscheidung über den Zeitpunkt der Durchführung den Studierenden zu überlassen 
Beispiele für einmalig durchgeführte Projekte des Forschenden Lernens, die deren grundsätzliche Möglichkeiten und Schwierigkeiten zeigen, werden in der Literatur in großer Fülle berichtet (z. B. in den Sammlungen von Huber, Hellmer \& Schneider, 2009; Huber, Kröger \& Schelhowe, 2013; Lepp \& Niederdrenk-Felgner, 2014; Mieg \& Lehmann, 2017; Kaufmann, Satilmis \& Mieg, 2018). Verbreitet sind Weiterentwicklungen aus Veranstaltungen der „Lehrforschung“ (zur Vermittlung von Methoden), etwa in den Sozialwissenschaften (eher in der Mitte des Studienganges), oder auch von Laborprojekten im Sinne von open-end labs, in denen Experimente mit offenem Ausgang ausgeführt werden, in den Naturwissenschaften (eher gegen Ende des Studienganges, evtl. vorbereitend zur Abschlussarbeit; z. B. Moerschbacher \& Rach, 2009). Inwieweit solche Vorhaben (anschließend) in Studiengängen verankert worden sind, ist eine andere Frage.

Die einmalige Verankerung von Forschungsnahem Lernen kann damit einhergehen, dass bestimmte Phasen des Studiums durch einen besonderen Schwerpunkt auf solche Veranstaltungen markiert werden. In der angelsächsischen College-Tradition liegt beispielsweise besonderer Nachdruck auf learning by research or inquiry in den First-Year-Programmen, die als Initiation in Studium und wissenschaftliches Arbeiten ausgestaltet sind (Healey, Jenkins \& Lea, 2014; für Deutschland Reinmann, 2018, S. 5). In deutschen Hochschulen wird Forschendes Lernen häufig (erst) in die Abschlussphase des Studiums gesetzt und mit der Vorbereitung auf eine Prüfungsarbeit oder deren Durchführung verbunden. Examens- und Diplomarbeiten wurden hier ja schon traditionell als auf eigener Forschung basiert verstanden; dergleichen auch für die Bachelorarbeit wenigstens zu ermöglichen, liegt nahe, während es für die angelsächsische Welt, die weithin für Bachelorabschlüsse eher nur die Summierung von ECTS-Punkten oder allenfalls Abschlussprüfungen in Form von Tests und mündlichen Prüfungen kannte, als Innovation behandelt wird (z. B. Healey, 2014).

Der Verankerung von Forschungsnahem Lernen - oder zumindest des Forschenden Lernens im engeren Sinne - an nur einer Stelle im Studium liegt, ob explizit oder nur implizit, eine Überlegung der Art zugrunde, dass es aus vielen Gründen nicht machbar oder (z. B. wegen des Zeitaufwandes oder der Berechtigung auch anderer Lernformen) nicht wünschenswert wäre, das ganze Studium oder große Teile davon als Forschungsnahes Lernen oder gar Forschendes Lernen im engeren Sinne auszugestalten, wohl aber die Studierenden wenigstens einmal, also exemplarisch, erfahren sollten, was es heißt, einen Forschungsprozess von Anfang bis Ende zu durchlaufen. 


\subsection{2 "Wiederholt"}

Die „wiederholte“ Verankerung von Forschungsnahem Lernen bedeutet: Im Studiengang sind mehrere Module vorgesehen, die möglichst so angelegt sind, dass jeweils Projekte des Forschungsnahen Lernens im engeren Sinne durchlaufen werden. Die Wiederholung ermöglicht, dass die Projekte sich im Studienverlauf in ihrer Komplexität steigern können. Hier lässt sich von einem „Spiralcurriculum“ (Taba, 1962) sprechen: Analog zur allgemeinen Didaktik, in der darunter verstanden wird, dass an sich komplizierte Inhalte in sehr reduzierter Form schon etwa in der Grundschule ein erstes Mal angesprochen werden können, um dann in wachsender Komplexität - „in wachsenden Ringen“ - auf höheren Schulstufen erneut angesprochen zu werden, stünde der Begriff hier dafür, dass Studierende zunächst einmal in vor allem im methodischen Anspruch sehr vereinfachten Projekten erfahren, was es heißen kann, selbst zu forschen und zu entwickeln, später aber noch ein- oder mehrmals anspruchsvollere Forschungsvorhaben ausführen.

Beispiele hierfür finden sich an Hochschulen, in denen schon in der Studieneingangsphase Forschendes Lernen praktiziert wird - in kleinen Projekten, die notwendigerweise relativ einfach und explorativ sind, aber ansatzweise den Forschungszyklus durchlaufen, woran dann im Verlauf des Studiums weitere Projekte anschließen können (z. B. das „Leuphana-Semester" an der Universität Lüneburg (Verfürth, 2019) oder das Einführungsmodul Maschinenbau der Technischen Universität Darmstadt (TU Darmstadt, Fachbereich Maschinenbau, 2019; Hampe, 2002). Ein elaboriertes Beispiel bietet das Projekt „Spiralcurriculum Forschendes Studieren in lehramtsbezogenen Studiengängen " an der Universität Bremen, das die für diesen Studiengang charakteristischen Praxisphasen besonders berücksichtigt.

\subsection{3 "Sequenziell"}

Von einem sequenziellen Aufbau von Forschungsnahem Lernen kann bei Studiengängen gesprochen werden, die von einer Reihenfolge von Modulen durchzogen werden, die zwar alle insgesamt am Forschungsnahen Lernen orientiert sind, in denen aber nicht jedes Modul den ganzen Zyklus des Forschens vorsieht, sondern jeweils unterschiedliche Elemente oder „Phasen“ (etwa Orientierung/Recherchen, Methodenerarbeitung/Übung, Durchführung/Präsentation) besonders akzentuiert werden.

Als ein Modell dafür ist das „Zürcher Framework zur Verknüpfung von Lehre und Forschung" zu betrachten (Tremp \& Hildbrand, 2012). Es geht von den bekannten Phasen des Forschungsprozesses aus, mit denen zugleich jeweils bestimmte Kompetenzen (für die Meisterung der entsprechenden Forschungsaufgaben - Recherche, Untersuchung, Präsentation ...) aufzubauen sind, die sich ihrerseits in spezifischen 
Produkten ausweisen (z. B. Thesenpapier, Forschungsübersicht, Laborjournal). Es zeigt auch auf, welche Veranstaltungsformen besonders geeignet sind, diese Kompetenzen zu vermitteln (die Vorlesung z. B. für die Forschungsübersicht, aber auch die Selbstvorstellung des Lehrenden mit seiner Forschung als Modell). All dieses, in eine Sequenz gebracht, die den Studiengang durchzieht, kann dahin führen, dass in der Bachelor-, spätestens aber in der Masterthesis ein eigenes Forschungsprojekt, das dann alle diese Elemente enthält, durchgeführt werden kann. Die Überschrift „Forschungsorientiert“ (Tremp \& Hildbrand, 2012) trifft das Konzept genau; es bezeichnet sowohl seine Stärke als auch seine Begrenzung: Das ganze Studium wird auf Forschung hin orientiert, die aber als eigene und damit vor allem auch mit einer eigenen Fragestellung erst am Schluss des Studiums ausgeübt wird. Bis dahin werden einzelne der dafür nötigen Kompetenzen wissenschaftlicher Arbeit den Zielen, Themen und Formaten der vorgesehenen Lehrveranstaltungen folgend entwickelt. Die von Huber (2014) oder Reinmann (2017) nebeneinander gestellten Typen des Forschungsnahen Lernens - Forschungsbasiertes Lernen/Forschung verstehen, Forschungsorientiertes Lernen/Forschung einüben, Forschendes Lernen/ Selbst-Forschen - werden hier nacheinander in eine Reihenfolge gesetzt. ${ }^{1}$ Eine solche Umwandlung von Typen in Stufen scheint übrigens nahezuliegen - sie findet sich zum Beispiel auch in dem bekannten "Research Skill Development Framework“ (Willison \& O’Regan, 2007; Fung, 2017). Eine ganz konsequente Sequenz wird aus der University of Indiana berichtet: „Students start as consumers of knowledge and move toward knowledge producers in eight steps [...]" (Dekker \& Wolff, 2016, S. 5).

Der Ausgangspunkt solcher Vorschläge, die wichtigsten Elemente des Forschungsprozesses auf eine Reihe verschiedener Veranstaltungen zu verteilen, ist die Überlegung, dass Forschendes Lernen in seiner idealen Form (als Format, in dem der gesamte Forschungsprozess durchlaufen wird) zu voraussetzungsreich sei; insbesondere würden fachliche Vorkenntnisse, vor allem aber methodische und soziale Kompetenzen benötigt, die nicht in ein und demselben Akt eingeholt werden könnten. Diese Sorge ist einerseits berechtigt, vor allem dann, wenn, wie in manchen Hochschulen Vorschrift, Module nicht mehr als ein Semester umfassen und auch nicht miteinander verkoppelt werden dürfen und der zeitliche Rahmen daher nicht reicht. Um ihr gerecht zu werden, müssten für Projekte des Forschenden Lernens im engeren Sinne Module vorgesehen werden, die sich über zwei Semester, einschließlich der dazwischen liegenden vorlesungsfreien Zeit für eventuelle Untersuchungen im Feld, in Archiven oder Laboren erstrecken und vielleicht auch von Methodenkursen oder Ähnlichem flankiert werden können.

1 So suggeriert es m.E. der Text; laut Tremp (mündlich) ist eine zeitliche Sequenzierung allerdings nicht zwingend. 
Andererseits bergen solche Verteilungsmodelle die Gefahr, dass eben auch die Erfahrung des ganzen Arbeitsbogens eines Forschungsprozesses zerteilt, die einzelnen Schritte bzw. Kompetenzen an ganz verschiedenen Inhalten geübt werden, das Bewusstsein des Zusammenhangs zwischen ihnen nicht entsteht, bevor günstigenfalls die Abschlussarbeit mit einem solchen Projekt verbunden wird. Erst mit diesem kommen dann eigene Fragestellungen und Selbstständigkeit zum Tragen; die vorausgehenden Veranstaltungen mit ihren Schwerpunkten auf Recherche oder Methodeneinführung oder Laborpraktika könnten ungeachtet ihrer verbalen Zuschreibung zum Forschungsnahen Lernen ziemliche Ähnlichkeit mit herkömmlichen lehrendenzentrierten Seminaren oder Übungen haben.

Lässt sich dieser Gefahr begegnen? Wollte man die verschiedenen Veranstaltungen unter den Bogen eines Forschungsprozesses, von einer übergreifenden Fragestellung geleitet, stellen, müsste man sie in diesem Sinne markieren, in eine verbindliche Reihenfolge bringen und für jeweils eine Kohorte von Studierenden die Teilnahme an dieser Sequenz durch die Semester hindurch obligatorisch machen (siehe Abschnitt 4.2).

\subsection{4 "Studienbegleitend"}

Eine Alternative wäre, die Reflexion der Zusammenhänge der verschiedenen Veranstaltungen mit einem Forschungsprozess bzw. einer Forschungsfrage in einer das Studium durchgehend begleitenden Aktivität zu beheimaten: in einer gleich anfangs gebildeten und fortdauernden Lerngruppe oder in einem ebenfalls kontinuierlichen Tutorium oder Mentoring-Programm oder in einem über die ganze Länge des Studiums gestreckten Modul.

Es mag an dieser Stelle von Interesse sein, zu sehen, dass man auch in englischen und US-amerikanischen Hochschulen, die den deutschen in der Förderung von „Research-Based Learning“ (RBL) bzw. „Undergraduate Research“ weit voraus sind, ähnliche Gedanken hegt. Eine Auswertung der Fülle von Literatur dazu ist hier nicht möglich. Als Beispiel sei Dilly Fung herangezogen, die vieles daraus für ein „Connected programme design“ zusammenfasst (Fung, 2017, Kap. 4.). Mit dem Ziel „a related sequence for research and enquiry, so that students steadily build up their abilities and confidence" nennt sie zehn mögliche Ansätze:

1. Eine Sequenz von forschungsorientierten Modulen, die sich durch den Studiengang zieht, möglichst einem übergreifenden Problem gilt und als eine zusammenhängende Lerngeschichte („learning narrative“) berichtet und reflektiert werden kann.

2. Eine Sequenz von verzweigten Modulen: dasselbe, aber mit mehr Wahlmöglichkeiten, auch inhaltlich. 
3. Ein einziges Modul, das in seinen Teilen oder Abschnitten ebenfalls, begleitend zu anderen Modulen, vom Anfang bis zum Ende eines Studienganges führt (abzubilden in einem Portfolio).

4. Studiengangbegleitende feste Gruppen für gemeinsame Arbeiten

5. Orientierung eines ganzen Studiengangs auf ein Abschlussereignis, zum Beispiel eine Forschungskonferenz, hin.

6. Ein das letzte Jahr krönendes Abschlussmodul (capstone module), für das die Studierenden alles Wissen zu einem komplexen Problem noch einmal aktivieren.

7. Eine Kern-Prüfungsleistung, die sich durch verschiedene Module hindurch aufbaut, beispielsweise ein reflexives Portfolio.

8. Ein Online-Begleitstrang zum Präsenzstudium, der strukturierte Bezüge zu Praxis, Forschung und Interdisziplinarität bietet.

9. Die Gewährleistung (im Studienangebot), dass verbindende Themen regelmäßig wieder angesprochen und weiterbearbeitet werden - als eine Art Leitmotiv.

10. Unterstützung des ganzen Studiums durch ein Tutorium als Reflexions- (oder Supervisions-)Gruppe.

Darin entsprechen die Vorschläge eins und zwei den in Abschnitt 3.2.3 besprochenen. Die Vorschläge drei, vier, zehn und, je nachdem, auch acht stellen die gewünschte Kohärenz durch studienbegleitende Vorkehrungen her. Inwieweit sie auf deutsche Hochschulen übertragbar wären, ist unterschiedlich fraglich. Die gewichtigste Implikation aller Vorschläge bei Fung ist aber, dass sie die durchgehende Teilnahme der Studierenden an diesen Maßnahmen für obligatorisch erklärt (Fung, 2017, S. 55). Das liegt zwar in der Konsequenz des Strebens nach curricularer Verankerung des Forschungsnahen Lernens, stößt aber meines Erachtens auf grundsätzliche Bedenken (siehe Abschnitt 4.2) und auch den Einwand, dass eine etwa zu Studienanfang entwickelte Forschungsidee und -fragestellung sich im weiteren Studium nicht nur sehr leicht verändern kann, sondern auch verändern können sollte. Insofern bleibt aus meiner Sicht doch nur das gute Ausweisen einer solchen Strecke im Studiengang und eine mit guten Gründen stark gemachte Empfehlung, ihr zu folgen.

\subsubsection{Verankerung des Forschungsnahen Lernens in Prüfungsordnungen und Prüfungspraxis}

Die curriculare Verankerung von Forschungsnahem Lernen ist erst mit der Berücksichtigung in Prüfungsordnungen und in der Prüfungspraxis vollständig. Sofern zu Veranstaltungen bzw. Projekten diesen Formates überhaupt Prüfungen stattfinden müssen - immerhin ist es denkbar, sie davon auszunehmen -, müssen die Formen, die dafür vorgesehen werden, einerseits hinreichend komplex sein, um die verschiedenen Kompetenzen zu erfassen, und andererseits hinreichend offen 
und vielfältig, um der Individualität der Leistungen, die aus Forschungsnahem Lernen hervorgehen können, gerecht zu werden (Huber, 2008; Reis \& Ruschin, 2008; Reinmann, 2017). Standardisierte Abfragen von bloßen Kenntnissen leisten das jedenfalls nicht; sie trotzdem einzusetzen, würde die Ziele des Forschungsnahen Lernens entwerten. Diesen entspräche es am besten, wenn Produkte, wie sie in wissenschaftlichen Prozessen normalerweise hervorgebracht werden (Ergebnisdarstellungen, Arbeitsberichte, Rezensionen, evtl. auch Materialiensammlungen) als Prüfungsleistungen genutzt werden und allenfalls noch einmal eine mündliche Verteidigung (Disputation) veranstaltet und bewertet wird.

\section{$4 \quad$ Grundsätzliche Fragen}

Über die curriculare Verankerung des Forschungsnahen Lernens entscheidet nicht der oder die einzelne Lehrende, sondern ein Kollektiv, ein Organ des Studiengangs, des Fachbereichs oder der Hochschulleitung, in dem einzelne Lehrende bestenfalls mitwirken können. Dieses bestimmt den Rahmen (bezüglich Inhaltsbindung, Pflichtcharakter, ECTS-Gewicht, Prüfungen, Zeitpunkten/Dauer und Ressourcen) für die individuellen Ausgestaltungen des Forschungsnahen Lernens auf der Mikroebene (Lübcke, Reinmann \& Heudorfer, 2017). Bei diesen Entscheidungen und der Wahl zwischen den oben aufgeführten Mustern stellen sich also grundsätzliche Fragen, deren Beantwortung zugleich die Ausgangsbedingungen für Forschungsnahes Lernen auf der Seite der Studierenden (Motivation, Wissensvoraussetzungen, Zeiteinsatz) stark beeinflusst (Reinmann, 2018, S. 6 ff.). Wenigstens die für unser Thema wichtigsten sollen hier, wenngleich nur kurz, angesprochen werden.

\subsection{Zeitrahmen}

Welchen Raum soll Forschungsnahes Lernen der einen oder anderen Form im Studiengang insgesamt einnehmen? Soll es ihn durchgehend bestimmen, einmalig/ exemplarisch erfahren werden können, wiederholt/leitmotivisch auftauchen? Viele Varianten sind da möglich. Die Entscheidung hängt zum einen von den Gründen und Zielen ab. Ist „Bildung durch Wissenschaft“ vorrangig, bietet Forschendes Lernen in großer Tiefe und Breite das größte Potenzial. Geht es in erster Linie um „employability“, wählte man Formen, welche die Aneignung von Schlüsselqualifikationen begünstigen. Die Dauer der einzelnen Projekte steht vermutlich im umgekehrten Verhältnis zu deren Häufigkeit: Die Konzentration auf ein einma- 
liges/exemplarisches Projekt mit dem ganzen Forschungszyklus verlangt einen größeren zeitlichen Rahmen als Veranstaltungen zu einzelnen seiner Etappen. Die Zeitpunkte im Studienverlauf hängen ebenfalls mit den jeweiligen Formen und deren Funktionen zusammen, wie die Muster oben zeigen.

Forschungsnahes Lernen zum allein geltenden Prinzip zu machen, wäre allerdings meines Erachtens fragwürdig - es ist nicht für alle Ziele gleich vorteilhaft. Neben den Studienzielen, die am besten durch Forschungsnahes Lernen angestrebt werden, stehen andere - auch berechtigte - für die jeweils andere Lernformen günstiger zu sein scheinen, zum Beispiel direkte Instruktion und gezieltes Üben für das Aneignen von Stoffkenntnissen und Fertigkeiten. Zudem ist meines Wissens bisher die Annahme nicht ausgeräumt, dass Forschungsnahes Lernen und besonders das Forschende Lernen im engeren Sinne nicht für alle Studierenden gleichermaßen geeignet ist (Wulf, 2017). Insofern wäre auch ein Konzept wie das der Universität Maastricht - Problem-based Learning als durchgehendes Prinzip für alle, zusätzlich Research-based Learning für ausgewählte Fortgeschrittene (Bastiaens, van Tilburg \& van Merriënboer, 2017) -, so eindrucksvoll es ist, noch einmal kritisch zu hinterfragen. Einstweilen gilt aus meiner Sicht eher die Maxime: Zwar sollten möglichst alle einmal damit Erfahrungen gemacht haben, aber im Übrigen müssen sie sich je nach ihren individuellen Lerndispositionen auch für andere Lernformen entscheiden können.

\subsection{Verpflichtungsgrad}

Damit ist erneut die oben schon berührte Frage angesprochen, ob und inwieweit Forschungsnahes Lernen für alle Studierenden eines Studiengangs obligatorisch gemacht werden sollte. Es gibt viele Gründe, sie zu bejahen: die Sorge, dass sonst viele Studierende davor auswichen, weil sie etwa das Risiko scheuen, den Zeitaufwand zu hoch, das ganze Engagement zu unkalkulierbar finden oder von sich aus ein zu geringes Interesse entwickelt haben und das Studium nur als Instrument für einen Abschluss möglichst ökonomisch bewältigen wollen - oder weil sie fürchten, dort, wo es um eine Art von Sequenz geht, schließlich, Stationen auf dem Weg zu übersehen und zu verpassen. Dagegen stehen Gründe, die Verpflichtung zu verneinen bzw. auf höchstens eine, dann möglichst exemplarische, Wahlpflichtveranstaltung zu beschränken: das Konzept eines Studiums mit möglichst großen Wahlfreiheiten im Allgemeinen und die lerntheoretische Begründung der hohen Bedeutung von Selbstbestimmung und Interesse (Deci \& Ryan, 1993 u. v. a.). 


\subsubsection{Zugangsbegrenzung}

Im Gegensatz zu einer Verpflichtung: Soll oder muss es eine Begrenzung des Zugangs $\mathrm{zu}$ (bestimmten) Projekten des Forschungsnahen Lernens geben? Je komplexer ein Projekt ist und je anspruchsvoller bezüglich der Ressourcen (z. B. Laborausstattung, Plätze im „Feld“), desto nötiger kann eine Begrenzung der Zahl durch ein Auswahlkriterium werden. Als Zugangsvoraussetzungen könnten eine besondere Motivation, wie auch immer festgestellt, oder vorausgehende Qualifizierungen (bereichsspezifische Kenntnisse, Forschungsmethoden) festgelegt werden - je nachdem, ob im Prinzip die Teilnahme an einem Projekt für jeden Pflicht oder Wahlpflicht ist oder völlig freigestellt.

\subsubsection{Fachspezifik}

Fachspezifische Unterschiede in Forschungsbegriff und Forschungspraxis prägen nicht nur die einzelnen Projekte des Forschungsnahen Lernens (siehe dazu Mieg in diesem Sammelband; Mieg \& Lehmann, 2017; Reinmann, 2018), sondern führen wahrscheinlich auch zu unterschiedlichen Formen der curricularen Verankerung, sowohl was Akzentuierungen der Typen als auch was Sequenzierungen angeht: Fächer, deren Curricula in ihrer Lehrstruktur - nach der Unterscheidung von Bernstein (Bernstein, 1977) - dem Kollektionscode (strikte Klassifikation der Inhalte nach innen, scharfe Abgrenzung nach außen) folgen, werden vermutlich dazu tendieren, Forschungsnahes Lernen, jedenfalls aber Forschendes Lernen im engeren Sinne erst später im Studium, auf einem Sockel von "Grundkenntnissen“, zu etablieren. Fächer mit integrativem Code (weichere Grenzen zwischen den Inhalten nach innen und nach außen) könnten schon früher eigene Versuche zulassen.

Selbstständiges Forschen (Experimentieren) im Labor zum Beispiel setzt offenbar voraus, dass die Studierenden im Vorfeld vielfältige Kenntnisse über die Arbeit im Labor und auch über Sicherheitsbestimmungen erworben haben, damit man sie dort überhaupt zulassen kann. In den Sozialwissenschaften dagegen scheinen auch für Studienanfänger kleine Erhebungen „vom Fleck weg“, wozu einfachere Methoden herangezogen oder entwickelt werden können, möglich, aus denen dann by doing gelernt werden kann. Daran könnte sich dann beispielsweise ein Spiralcurriculum anschließen. 


\section{$5 \quad$ Schlussbemerkung}

Curriculare Verankerung steht nicht am Anfang des Forschungsnahen Lernens in einer Hochschule, sondern setzt voraus, dass es schon Engagement und Aktivitäten gibt, die nach einer solchen Ordnung verlangen. Schon gar nicht darf sich die Förderung des Forschungsnahen Lernens lediglich in einer curricularen Verankerung erschöpfen. Für eine Förderung ist wichtiger, dass die Hochschule die Lehrenden - und für freie Projekte auch die Studierenden - zu Forschungsnahem Lehren ermuntert (Anreize) und befähigt (Fortbildungsangebote), Austausch zwischen ihnen stiftet, Hilfen zur Verfügung stellt (siehe z. B. Langemeyer, 2018), Experimente unterstützt (Förderung), Freiräume in ihren Studien- und Prüfungsordnungen dafür lässt und Leistungen würdigt (z. B. durch Lehrpreise oder Ausstellungen). In einem solchen Klima kann eine curriculare Verankerung dann ein Gerüst für Lehrende und Studierende statt einer Klammer bieten und stetige Anstöße statt pflichtgemäße Routine hervorbringen.

\section{Literatur}

Bastiaens, E., van Tilburg, J. \& van Merriënboer, J. (2017). Research-based learning: Case studies from Maastricht University. Cham: Springer.

Bernstein, B. (1977). Über Klassifikation und Rahmung pädagogisch vermittelten Wissens. In B. Bernstein (Hrsg.), Beiträge zu einer Theorie des pädagogischen Prozesses (S. 125-161). Frankfurt: Suhrkamp.

Bundesassistentenkonferenz (BAK) (1970). Forschendes Lernen - Wissenschaftliches Prüfen (Schriften der BAK, 5. Neudruck) Bielefeld: UVW.

Deci, E. L. \& Ryan, R. M. (1993). Die Selbstbestimmungstheorie der Motivation und ihre Bedeutung für die Pädagogik. Zeitschrift für Pädagogik, 39(2), S. 223-238.

Dekker, H. \& Wolff, S. W. (2016). Re-Inventing Research-Based Teaching and Learning: Paper prepared for presentation at the meeting of the European Forum for Enhanced Collaboration in Teaching of the European University Association in Brussels on 5 December 2016. Verfügbar unter https://www.researchgate.net/publication/311537070_Re-Inventing_Research-Based_Teaching_and_Learning [12.08.2019].

Fung, D. (2017). A Connected Curriculum for Higher Education. London: UCL

Hampe, M. (2002). Einführung in den Maschinenbau: Ein Projektkurs für Erstsemester. Das Hochschulwesen, 50(6), S. 228-234.

Healey. M. (2014). Integrating undergraduate research into the curriculum: International perspectives on capstone and final-year project. CUR Quarterly 34(4), S.26-32.

Healey, M., Jenkins, A. \& Lea, J. (2014). Developing research-based currciula in college-based highger education. York: The Higher Education Academy. Verfügbar unter https:// 
www.heacademy.ac.uk/system/files/resources/developing_research-based_curricula_in_cbhe_14.pdf [12.08.2019].

Huber, L. (2008). ,Kompetenzen` prüfen? In S. Dany, B. Szczyrba \& J. Wildt (Hrsg.), Prüfungen auf die Agenda! Hochschuldidaktische Perspektiven auf Reformen im Prüfungswesen (Blickpunkt Hochschuldidaktik, 118, S. 12-26). Bielefeld: Bertelsmann.

Huber, L. (2014). Forschungsbasiertes, Forschungsorientiertes, Forschendes Lernen: Alles dasselbe? Ein Plädoyer für eine Verständigung über Begriffe und Unterscheidungen im Feld forschungsnahen Lehrens und Lernens. Das Hochschulwesen, 62(1 \& 2), S.32-39.

Huber, L., Hellmer, J. \& Schneider, F. (Hrsg) (2009). Forschendes Lernen im Studium: Aktuelle Konzepte und Erfahrungen. Bielefeld: UVW.

Huber L., Kröger, M. \& Schelhowe, H. (Hrsg.) (2013). Forschendes Lernen als Profilmerkmal einer Universität: Beispiele aus der Universität Bremen. Bielefeld: UVW

Kaufmann, M. E., Satilmis, A. \& Mieg, H. A. (Hrsg.) (2018). Forschendes Lernen in den Geisteswissenschaften: Einblicke in Konzepte, Praktiken und Perspektiven hermeneutischer Fächer. Heidelberg: Springer SV

Langemeyer, I. (2018). Lehrvideos Forschendes Lernen. Verfügbar unter http://lehr-lernforschung.org/?page_id=9 [12.08.2019].

Lepp, S. \& Niederdrenk-Felgner, C. (Hrsg.) (2014). Forschendes Lernen initiieren, umsetzen und reflektieren. Bielefeld: UVW.

Lübcke, E., Reinmann, G. \& Heudorfer, A. (2017). Entwicklung eines Instruments zur Analyse forschenden Lernens. Zeitschrift für Hochschulentwicklung, 12(3), S.2-24.

Mieg, H. A. \& Lehmann, J. (Hrsg.) (2017). Forschendes Lernen: Wie die Lehre in Universität und Fachhochschule erneuert werden kann. Frankfurt: Campus.

Moerschbacher, B. \& Rach, J. (2009). Das „Projektmodul: Ein Rahmen für Forschendes Lernen in den Biowissenschaften“. L. Huber, J. Hellmer \& F. Schneider (Hrsg.), Forschendes Lernen im Studium: Aktuelle Konzepte und Erfahrungen (S. 169-178). Bielefeld: UVW.

Reinmann, G. (2017). Prüfungen und Forschendes Lernen: Wie die Lehre in Universität und Fachhochschule erneuert werden kann. In H. A. Mieg \& J. Lehmann (Hrsg.), Forschendes Lernen: Programmatik und Praxis (S.115-128). Frankfurt: Campus.

Reinmann, G. (2018). Heuristiken für die Hochschullehre zur Förderung forschenden Lernens. Hamburg: HUL Typoskript.

Reinmann, G. (2018). Lernen durch Forschung - aber welche? In N. Neuber, W. Paravicini \& M. Stein (Hrsg.), Forschendes Lernen - the wider view. Münster: WTM

Reis O. \& Ruschin, S. (2008). Kompetenzorientiert prüfen - Baustein eines gelungenen Paradigmenwechsels. In S. Dany, B. Szczyrba \& J. Wildt (Hrsg.), Prüfungen auf die Agenda! Hochschuldidaktische Perspektiven auf Reformen im Prüfungswesen, (Blickpunkt Hochschuldidaktik, 118, S. 45-57). Bielefeld: Bertelsmann.

Robinsohn, S. B. (1972). Bildungsreform als Revision des Curriculum und ein Strukturkonzept für Curriculumentwicklung. Neuwied: Luchterhand.

Stang, T. (2020). Formate forschungsnahen Lehrens und Lernens an Universitäten in Deutschland - eine empirische Untersuchung (Dissertation). Universität Bielefeld. DOI: 10.4119/ unibi/2941421

Taba, H. (1962). Curriculum Development: Theory and Practice. New York: Harcort

Tremp, P. \& Hildbrand, T. (2012). Forschungsorientiertes Studium - universitäre Lehre: Das „Zürcher Framework zur Verknüpfung von Lehre und Forschung“. In T. Brinker \& P. Tremp (Hrsg.), Einführung in die Studiengangentwicklung (Blickpunkt Hochschuldidaktik, 122, S. 101-116). Bielefeld: Bertelsmann 
Willison, J. \& O’Regan, K. (2007). Commonly known, commonly not known, totally unknown: A framework for students becoming researchers. Higher Education Research and Development 26(4), S.393-409.

Wulf, C. (2017). „From Teaching to Learning“ - Merkmale und Herausforderungen einer studierendenzentrierten Lernkultur. In H. A. Mieg \& J. Lehmann (Hrsg.), Forschendes Lernen: Wie die Lehre in Universität und Fachhochschule erneuert werden kann (S.66-75). Frankfurt: Campus.

\section{Weitere Quellen}

RWTH Aachen University (Hrsg.) (2019). Das Undergraduate Research Opportunities-Programm (UROP). Verfügbar unter http://www.rwth-aachen.de/cms/root/Forschung/ Angebote-fuer-Forschende/Angebote-fuer-Studierende/ rmt/UROP/ [12.08.2019].

TU Darmstadt, Fachbereich Maschinenbau (Hrsg.) (2019). Bachelorstudiengang Maschinenbau - Mechanical and Process Engineering (B. Sc.). Verfügbar unter https://www.maschinenbau. tu-darmstadt.de/media/maschinenbau/dokumente_2/studieren_1/neue_pruefungsordnungen_3__0_2014/Modulhandbuch_Bachelor_MPE_19_02_26.pdf [12.08.2019].

Universität Hohenheim (Hrsg.) (2019). Humboldt reloaded. Verfügbar unter https://humboldt-reloaded.uni-hohenheim.de [12.08.2019].

Verfürth, C. (2019). Leuphana Semeser am College. Verfügbar unter https://www.leuphana. de/college/studienmodell/leuphana-semester.html [12.08.2019].

\section{Zum Autor}

Prof. em. Dr. Dr. h. c. Ludwig Huber ${ }^{\dagger}$, ehemaliger Professor für Pädagogik (Wissenschaftsdidaktik), Fakultät Erziehungswissenschaften, und ehemaliger Wissenschaftlicher Leiter des Oberstufen-Kollegs, Universität Bielefeld. Arbeitsschwerpunkte: Hochschuldidaktik, besonders zu den Themen Forschendes Lernen, Scholarship of Teaching and Learning, Fachkulturen, Wissenschaftspropädeutik. 
Open Access Dieses Kapitel wird unter der Creative Commons Namensnennung 4.0 International Lizenz (http://creativecommons.org/licenses/by/4.0/deed.de) veröffentlicht, welche die Nutzung, Vervielfältigung, Bearbeitung, Verbreitung und Wiedergabe in jeglichem Medium und Format erlaubt, sofern Sie den/die ursprünglichen Autor(en) und die Quelle ordnungsgemäß nennen, einen Link zur Creative Commons Lizenz beifügen und angeben, ob Änderungen vorgenommen wurden.

Die in diesem Kapitel enthaltenen Bilder und sonstiges Drittmaterial unterliegen ebenfalls der genannten Creative Commons Lizenz, sofern sich aus der Abbildungslegende nichts anderes ergibt. Sofern das betreffende Material nicht unter der genannten Creative Commons Lizenz steht und die betreffende Handlung nicht nach gesetzlichen Vorschriften erlaubt ist, ist für die oben aufgeführten Weiterverwendungen des Materials die Einwilligung des jeweiligen Rechteinhabers einzuholen.

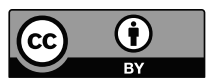

